\title{
Correlation of myomir-206 and proinflammatory cytokines (IL-16 and IL-17) in patients with rheumatoid arthritis
}

\author{
Amira Abo ElAtta ${ }^{1}$, Yasser Ali $^{1}$, Iman Bassyouni ${ }^{2}$, Roba Talaat ${ }^{1}$ \\ ${ }^{1}$ Molecular Biology Department, Genetic Engineering and Biotechnology Research Institute (GEBRI), University of Sadat City, Egypt \\ ${ }^{2}$ Department of Rheumatology and Rehabilitation, Faculty of Medicine, Cairo University, El-Kasr El-Aini Hospital, Egypt
}

\begin{abstract}
Introduction: Rheumatoid arthritis (RA) is a persistent autoimmune disease in which the activity of proinflammatory cytokines and the imbalance, related to the inflammatory process, between elements of bone tissue remodeling such as osteoclasts and osteoblasts play a key role in development of erosions and bone destruction. MicroRNAs are important regulators of skeletal remodeling and are involved in RA pathogenesis. Myomir-206 (miR-206) is unrivalled among the myomirRs, where it is expressed in skeletal muscle and either absent or minimally expressed in other tissues

Material and methods: This study was designed to analyze the miR-206 expression pattern in peripheral blood mononuclear cells (PBMCs) using quantitative real time polymerase chain reaction and its correlation with IL-16/IL-17 proinflammatory cytokines in two groups -20 healthy individuals and 30 patients with RA.

Results: Elevated expression of miR-206 was observed in RA patients compared with healthy controls $(p<0.001)$. A significant increase in both IL-17 and IL-16 serum levels was found in the RA group ( $p<0.01$ and $p<0.05$; respectively) compared to the control group. miR-206 expression level and IL-17 production were directly positively correlated $(r=0.491 ; p<0.01)$. ROC analysis of miR-206 showed a cutoff value of 2.7 with $70 \%$ sensitivity, $85 \%$ specificity, and the area under the curve was $0.802(p<0.001)$ with the $95 \%$ confidence interval from 0.676 to 0.927

Conclusions: Taken together, our results indicate the importance of miR-206 expression in patients with RA, as a potential new biomarker that affects bone loss/deformity and its collaborative role with proinflammatory cytokines such as IL-16 and IL-17 in RA bone metabolism. Particular interest should be given to further research to determine the contribution of expression of miR-206 in RA pathogenesis
\end{abstract}

Key words: rheumatoid arthritis, proinflammatory cytokines, interleukin, myomir-206.

\section{Introduction}

Rheumatoid arthritis (RA) is a chronic systemic autoimmune/inflammatory disease that is characterized by synovial hyperplasia and vascularization, and progressive destruction of cartilage and bone [1]. Activated T cells, predominantly CD4+ T cells infiltrating the synovium, are responsible for these RA-associated changes, and this is the reason why RA is considered as a Th-1-associated disease. T cells' activation, their migration as well as production of several inflammatory cytokines such as interleu- kin 2 (IL-2), IL-6, IL-16, IL-17, interferon (IFN- $\gamma$ ) and tumor necrosis factor (TNF- $\alpha$ ) are important for the initiation and perpetuation of RA-related pathology [2, 3].

The proinflammatory cytokine $\mathrm{IL}-16$ is a lymphocyte chemoattractant factor secreted by CD4+, CD8+ T lymphocytes, monocytes, epithelial cells and fibroblasts [3]. After binding to its receptor, IL-16 stimulates CD4+ T cell and monocyte migration to inflamed sites and also promotes proliferation of these cells [4]. IL-16 is generally considered to play a decisive role in both immune and inflammatory

\section{Address for correspondence:}

Roba Talaat, Molecular Biology Department, Genetic Engineering and Biotechnology Research Institute (GEBRI), University of Sadat City, Sadat City 11211, Egypt, e-mail: robamtalaat@yahoo.com

Submitted: 27.08.2018; Accepted: 15.04.2019 
responses. This cytokine plays a special role not only in the development of arthritis but also systemic RA manifestations; it also participates in the transformation of acute inflammation into a chronic process [5, 6].

IL-17 inflammatory cytokine plays a key role in the propagation of joint inflammation, cartilage destruction, and bone erosion $[7,8]$. It promotes recruitment of both neutrophils and monocytes by means of inducing various chemokines and is associated with severity of synovial inflammation of RA patients. Cho et al. [9] reported that IL-17 can induce the production of IL-16 in RA patients.

MicroRNAs (miRNAs) are an abundant class of evolutionarily conserved, single-stranded RNA molecules that have emerged as important posttranscriptional regulators of gene expression [10]. Gene expression by miRNAs has recently been identified to be important for basic function of the immune system [10,11]. Moreover, miRNAs contribute to the terminal differentiation of mature lymphocytes [12]. Many miRNAs are expressed in a tissue-specific manner, suggesting a role of the miRNAs in the specification of the tissue during differentiation. miR-206, a vertebrate-specific miRNA, is called a skeletal muscle specific myomiR $[12,13]$. It shares the same seed sequence and at least some targets with miR-1, which also belongs to the myomiR family along with miR-133a, miR-133b, miR-208a, miR-208b and miR-499 [14]. miR-206 is unique among the myomiR family in that it is specifically expressed in skeletal muscle, being absent or expressed at relatively low levels in other tissues. It is being intensively studied in several conditions such as skeletal muscle development, heart failure [14], various types of cancers and other diseases.

A positive correlation in expression of miR-133b/ miR-206 and IL-17 was found. miR-206 and miR-133b are co-transcribed together with IL-17 when IL-23 is used, which is specific for T-lymphocytes since these miRNAs were clustered nearly $45 \mathrm{~kb}$ upstream of the IL-17a/f locus. It appears that miR-206 regulation of IL-17 is context dependent [15]. Proinflammatory cytokines such as TNF- $\alpha, I L-1, I L-6$, and IL-17 tend to be osteoclastogenic. Knowing that altered miR-206 expression has been observed in several muscle disorders, we postulated that miRNAs might participate with some inflammatory cytokines in the pathogenesis of RA. To address this hypothesis, we studied the correlation between miR-206 expression and other circulatory levels of IL-16 and IL-17 proinflammatory cytokines in RA patients.

\section{Material and methods}

Thirty patients with RA diagnosed according to current American College of Rheumatology/European
League Against Rheumatism (ACR/EULAR) criteria were included in the study. Demographic and clinical characteristics were collected through a structured interview and physical examination. Patients with infection, malignancy as well as with other co-existing autoimmune diseases were excluded from this study.

Disease activity was assessed using the Disease Activity Score 28 (DAS28). Laboratory tests such as rheumatoid factor (RF) and the erythrocyte sedimentation rate (ESR) and other routine biochemistry tests were performed. Functional ability was assessed using the modified version of the Health Assessment Questionnaire (mHAQ) [16]. Radiographs of the hands and feet with the evaluation of erosive disease using scoring based on the Sharp score (ShS) was done by standard radiographs for all studied patients. At least one definite erosive change on any of the hands or feet radiographs was sufficient for inclusion of such a patient in the "erosive" group. Twenty healthy subjects with no previous history of autoimmune disease were included in the control group. The present study was performed according to the Ministry of Health, Health and Human Ethical Clearance Committee guidelines for Clinical Research and with approval of Cairo University local Ethics Committee. From all study subjects (patients and healthy controls) written consent was obtained.

\section{Measurement of miR-206 by quantitative real-time RT-PCR (qRT-PCR)}

Samples of $5 \mathrm{ml}$ of blood were withdrawn in ethylene diamine tetra acetic acid (EDTA) sterile tubes. Isolation of peripheral blood mononuclear cells (PBMCs) by Ficoll-Hypaque density gradient centrifugation, isolation of total RNA from freshly obtained PBMCs using the TRIzol isolation protocol (Thermo Fisher Scientific, Waltham, MA, USA) and qRT-PCR for the detection of Hs_miR-206 that was carried out in $25 \mu \mathrm{l}$ PCR reactions using the miScript SYBR Green PCR Kit and miScript Primer Assays (Qiagen) were performed as previously described [17].

\section{Detection of circulating interleukin 16 and 17}

Total concentrations of IL-16 and IL-17A were detected using commercial enzyme linked immunosorbent assay (ELISA) kits (R\&D Systems Inc., Minneapolis, MN) according to the manufacturer's recommendations. Absorbencies were measured at $450 \mathrm{~nm}$ using an ELISA plate reader (Sunrise, Tecan Group Ltd.). The ELISA reader-controlling software (Magellan 71, Tecan Group Ltd.) processes the digital data of raw absorbance values into a standard curve from which cytokine concentrations of unknown samples can be derived directly. Results - 
Table I. Demographic and laboratory characteristics of patients with rheumatoid arthritis (RA)

\begin{tabular}{|c|c|}
\hline Parameter & Mean \pm SD \\
\hline Age (years) & $39.10 \pm 10.43$ \\
\hline Female/Male & $27 / 3$ \\
\hline Disease duration (years) & $3.49 \pm 2.46$ \\
\hline Tender joints (of 28) & $8.53 \pm 6.75$ \\
\hline Swollen joints (of 28) & $4.03 \pm 4.45$ \\
\hline DAS-28 & $4.36 \pm 1.00$ \\
\hline $\mathrm{mHAQ}$ & $0.64 \pm 0.52$ \\
\hline Sharp score of erosions (0-170) & $37.26 \pm 33.95$ \\
\hline Parameter & $n(\%)$ \\
\hline \multicolumn{2}{|l|}{ Disease activity } \\
\hline Low (DAS28 < 3.2) & $4(13.3)$ \\
\hline Moderate $(3.2<$ DAS28< $<.1)$ & $20(66.7)$ \\
\hline High (DAS28 > 5.1) & $6(20)$ \\
\hline$R F+v e$ & $18(60)$ \\
\hline Subcutaneous nodules & $5(16.6)$ \\
\hline Extra-articular disease & $8(26.6)$ \\
\hline Laboratory parameters & Mean \pm SD \\
\hline ESR $(\mathrm{mm} / \mathrm{h})$ & $39.86 \pm 21.97$ \\
\hline Hemoglobin (g/dl) & $12.00 \pm 1.47$ \\
\hline $\operatorname{TLC}(\times 1000 / \mu l)$ & $8.02 \pm 3.412$ \\
\hline Platelets $(\times 1000 / \mu \mathrm{l})$ & $269.73 \pm 74.13$ \\
\hline Creatinine (mg/dl) & $0.72 \pm 0.30$ \\
\hline $\operatorname{ALT}(\mathrm{U} / \mathrm{I})$ & $21.20 \pm 17.42$ \\
\hline AST $(\mathrm{U} / \mathrm{l})$ & $22.03 \pm 9.99$ \\
\hline
\end{tabular}

ALT - alanine aminotransferase; AST - aspartate aminotransferase; DAS28 - Disease Activity Score 28; ESR - erythrocyte sedimentation rate; RF-rheumatoid factor; TLC - total leukocyte count.

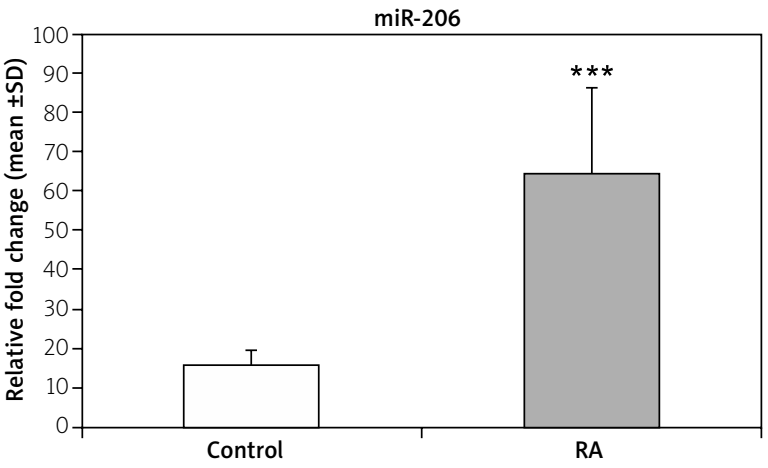

* denotes significant difference from healthy controls. ${ }^{* * *} p<0.001$.

Fig. 1. Relative fold change expression levels of miR-206 in rheumatoid arthritis (RA) and normal controls. cytokine concentration were expressed in picograms per milliliter (pg/ml).

\section{Statistical analysis}

All statistical analyses were performed using SPSS version 19 (SPSS, Inc., Chicago, IL). Data were statistically described in terms of mean \pm standard deviation $( \pm S D)$, and frequencies when appropriate. For the non-parametric tests the Spearman rank correlation was used, Student's t-test was used for comparison of numerical variables between the study groups for quantitative variables and also the one-way ANOVA test was used; $p$-value $<0.05$ was considered as significant.

\section{Results}

\section{Demographic and laboratory characteristics of RA patients and control group}

The 30 RA patients included 27 female and 3 males, F/M ratio $9: 1$ with age from 22 to 62 years (mean age $39.10 \pm 10.43$ years). The patients' age at the time of disease onset ranged from 18 to 60 years and disease duration ranged from 1 to 9 years. Healthy individuals in the control group were optimally matched in terms of gender and age (mean age of $28.28 \pm 8.77$ years). The descriptive data of the patients and healthy control are shown in Table I. Patients were classified as having active (low, moderate or high activity) or inactive disease on the basis of the DAS28.

\section{miR-206 expression levels in RA patients and control group}

The data presented in Figure 1 demonstrate a significant increase $(p<0.001)$ in the fold change expression levels of miR-206 in PBMCs of RA patients compared with the control group. A receiver operating characteristic (ROC) curve (Fig. 2) was constructed for the studied markers to estimate the best cut-off which could improve the specificity and sensitivity of miR-206 to differentiate between the studied groups. The ROC curve for the RA group vs. the control group showed a cutoff value of 2.75 , miR-206 with $70 \%$ sensitivity and $85 \%$ specificity, the area under the curve $0.802(p<0.001)$ with 95\% confidence interval (Cl): 0.676-0.927.

Figure 3 shows a significant increase of IL-16 $(p<0.05)$ and IL-17 $(p<0.01)$ serum level in RA patients compared with the control group. A positive correlation was found between miR-206 and IL-17 ( $r=0.491 ; p<0.01)$ (Fig. 4). No correlation was found between miR-206 and IL-16 concentration.

Disease activity of RA patients was classified into low, moderate and high depending on DAS28 values. 
There were no statistically significant correlation between high expression levels and low expression levels of miR-206 and disease activity, which is presented in Figure 5. There were no significant correlations between miR-206, IL-16 and IL-17 and clinical manifestation of RA.

\section{Discussion}

miRNAs are epigenetic regulatory mechanisms that impart broad and long-term changes in gene expression. They are able to modulate target gene translation via binding to the $3^{\prime}$-untranslated region (UTR) of miRNAs, thus affecting multiple protein-encoding genes at the posttranscriptional level, and they are implicated in the control of a wide range of biological functions $[18,19]$.

Documented data have shown that some miRNAs regulate bone homeostasis, including bone formation, resorption, remodeling, repair and bone-related diseases, by regulating the expression of certain cytokines and transcription factors [20]. miR-206 was originally found to be a specific miRNA for muscle differentiation under physiological conditions [21]. In muscular dystrophies, miR-206 is up regulated, as a form of "self-treating" significance. It has been recently discovered that miR-206 is also expressed in osteoblasts and its expression decreased during osteoblast differentiation. Overexpression of miR-206 inhibits osteoblast differentiation while the knockout of miR-206 promotes osteoblast differentiation [22]. miR-206 is referred to as a negative regulator of osteoblast differentiation [23]. Rheumatoid arthritis is a systemic, chronic inflammatory disease characterized by increase in bone resorption and impaired bone formation leading to joint destruction, deformity, and disability. Osteoblasts probably play a role in the pathogenesis of bone erosions in RA [24].

Patients with RA have alterations in their cellular miRNAs [25]. Dysregulation of miRNAs in effector cells in joint destruction such as PBMCs, T lymphocytes, synovial fibroblasts and osteoclasts was shown to contribute to inflammation, extracellular matrix degradation, and invasiveness of resident cells [26]. Concerning this idea, the role of miR-206 in regulation of osteoblast/osteoclast in RA as a bone-related disease needs to be fully elucidated; moreover there have been no documented articles that identify the role of miR-206 in RA patients till now.

In this study we used qRT-PCR to demonstrate the expression of miR-206 levels in PBMCs of RA patients compared with healthy controls. Our findings showed a highly significant increase in the expression levels of miR-206 in RA patients compared with the normal control group. Despite the absence of RA studies, there are some studies about miR-206 in other autoimmune diseases. In systemic lupus erythematosus (SLE) and der-

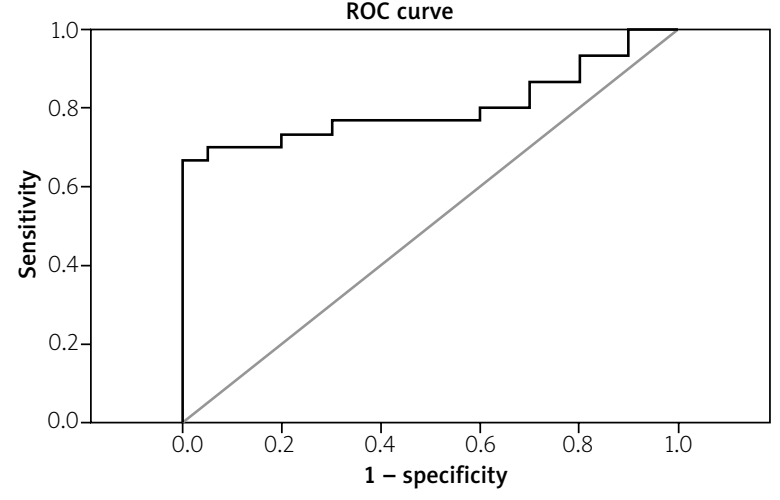

Fig. 2. Area under curve of receiver operating characteristic (ROC) of the miR-206 to discriminate RA patients from normal controls.
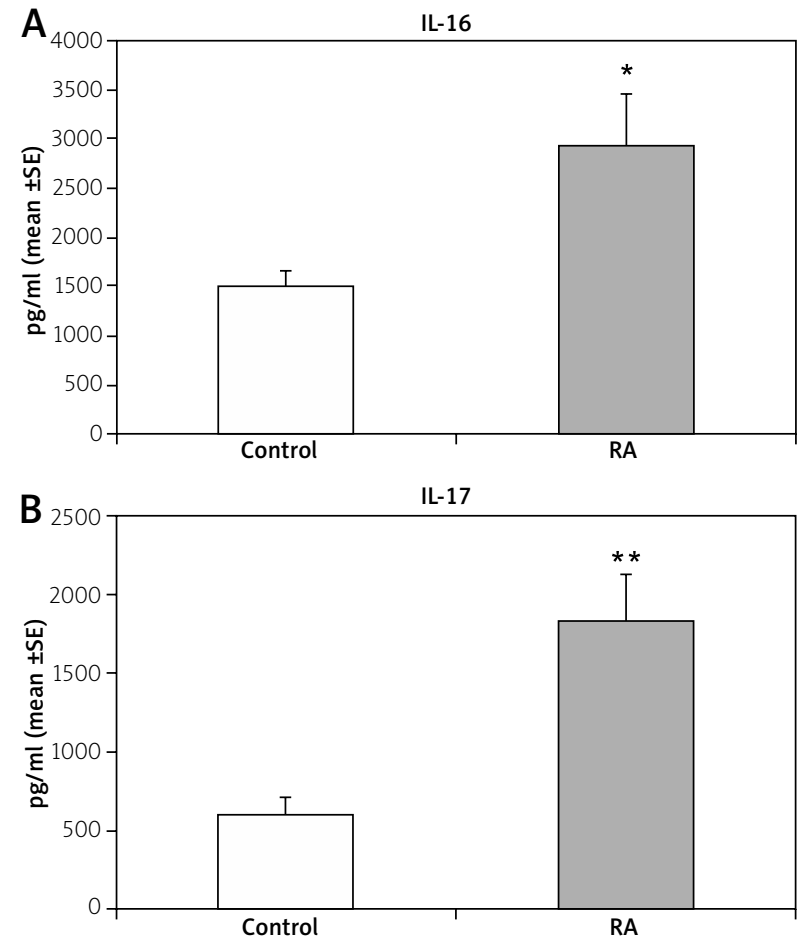

* denotes significant difference from healthy controls. ${ }^{* * *} p<0.001$.

Fig. 3. Interleukin 16 and interleukin 17 concentration in rheumatoid arthritis (RA) and normal controls.

matomyositis (DM) [27, 28], the expression of miR-206 in the PBMCs of patients was significantly decreased compared with that of healthy controls.

The osteoclast is a target of pro-inflammatory cytokines such as TNF- $\alpha, \mathrm{IL}-1, \mathrm{IL}-6$, and IL-17 and is an effector in initiating bone destruction and joint erosion in RA. This suggests that osteoclastogenesis should be carefully controlled to maintain normal bone remodeling or to reduce the progression of ectopic bone resorption and erosion in RA. Since the first demonstration that IL-17 is crucially involved in RA, scores of papers during the 


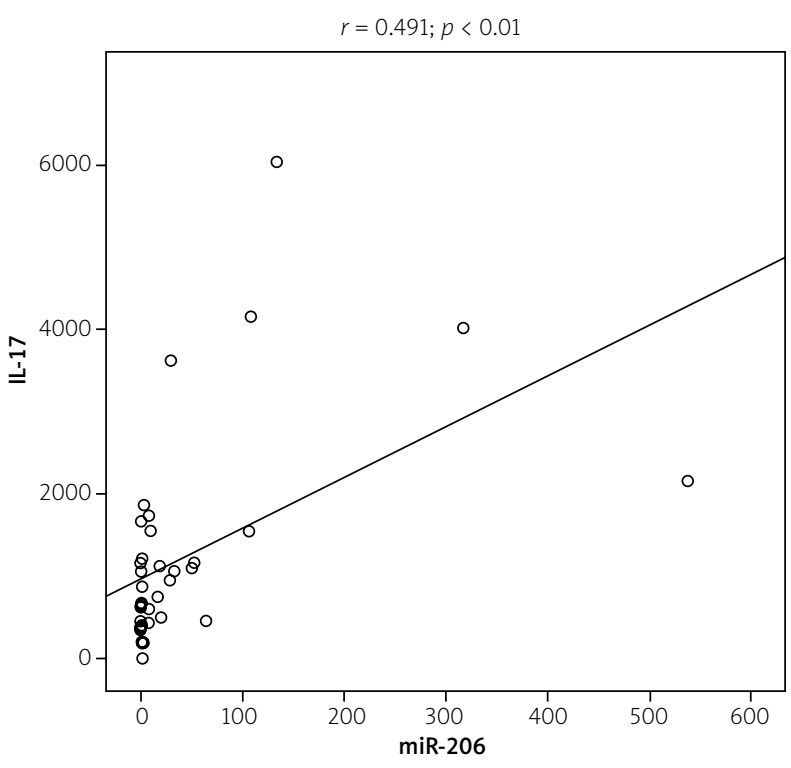

Fig. 4. Correlation between miR-206 and interleukin 17 in RA patients.

last decade have confirmed its role, and implication in RA. Our results showed that IL-17 was present at high levels in sera of RA patients; the majority of studies of IL-17 in RA have confirmed our data [29]. In accordance with Haas et al. [15] a positive correlation in expression of miR-206 and IL-17 production was found to confirm their contribution and interplaying role in RA pathology.

IL-17 stimulates the production of several inflammatory mediators, including IL-6, IL-8, granulocyte-macrophage colony stimulating factor (GM-CSF), and prostaglandin E2 (PGE2) in synoviocytes. IL-17 accelerates bone metabolism by stimulating osteogenic differentiation of mesenchymal stem cells and osteoblasts and promoting pro-osteoclastogenic molecules on these cells. It supports osteoclastogenesis dependent on the receptor activator of nuclear factor $\kappa$ and its ligand, RANK/RANKL, signaling pathway. It has been reported that IL-17 activity is synergistically increased with other pro-inflammatory cytokines and ultimately resulted in joint inflammation and damage [30].

One of these proinflammatory cytokines is $1 \mathrm{~L}-16$, which has been induced by IL-17 in RA patients. IL-17 and the specific ligand of TLR2 were shown to be the major stimuli that induced IL-16 expression and production in fibroblast-like synoviocytes (FLS) [9]. This might explain the elevation of both IL-16 and II-17 in our study. Blaschke et al. [31] demonstrated that IL-16 was detected at significantly higher levels in sera and synovial fluid (SF) of patients with RA in comparison to osteoarthritis (OA). Interesting contrary results were presented by Klimiuk et al. and in their study IL-16 acted as an im-

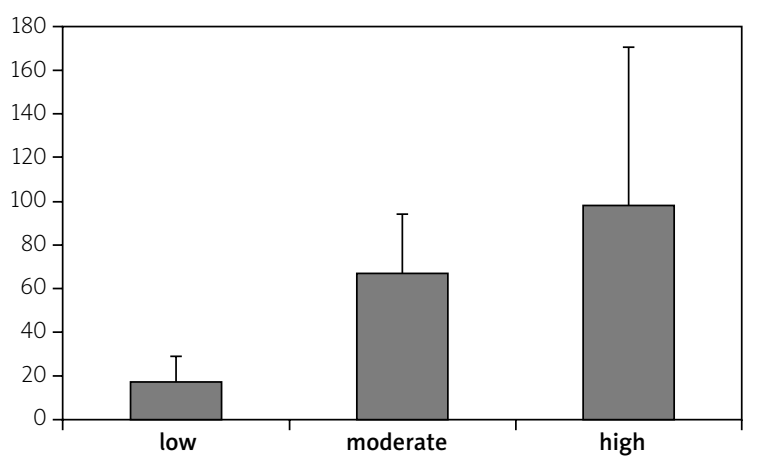

Fig. 5. Change in miR-206 expression in RA patients with different degrees of disease activity.

munomodulatory mediator and IL-16 administration in a murine model of RA decreased the production of IFN- $\gamma$, IL-1 $\beta$ and TNF- $\alpha$ [32]. What is worth emphasizing, in the literature there is no described research on the correlation between IL-16 and the expression of miR-206, which makes the present study promising in this topic.

\section{Conclusions}

Taking together, our results suggest that the elevated expression of miR-206, and proinflammatory cytokines (such as IL-16 and IL-17) might be involved in RA pathogenesis. A better understanding of the pathologic role of myomiRs such as miR-206 and their interplay with proinflammatory cytokines which influence bone metabolism will provide greater insight into the osteolytic process and ensure future development of therapies to ameliorate bone loss in RA. Thus, further investigations are warranted to explain this important aspect of RA pathophysiology.

The authors declare no conflict of interest.

\section{References}

1. Baillet A, Trocmé C, Berthier S. Synovial fluid proteomic fingerprint: S100A8, S100A9 and S100A12 proteins discriminate rheumatoid arthritis from other inflammatory joint diseases. Rheumatology 2010; 49: 671-682.

2. Choy EH, Panayi GS. Cytokine pathways and joint inflammation in rheumatoid arthritis. N Engl J Med 2001; 344: 907-916.

3. McInnes IB, Schett G. The pathogenesis of rheumatoid arthritis. N Engl J Med 2011; 365: 2205-2219.

4. Cruikshank WW, Kornfeld H, Center DM. Interlekin-16. J Leukoc Biol 2000; 67: 757-766.

5. Skundric DS, Zhou W, Cruikshank WW, Dai R. Increased levels of bioactive IL-16 correlate with disease activity during relapsing experimental autoimmune encephalomyelitis (EAE). J Autoimmun 2005; 25: 206-214. 
6. Srirangan S, Choy EH. The role of interleukin 6 in the pathophysiology of rheumatoid arthritis. Ther Adv Musculoskelet Dis 2010; 2: 247-256.

7. Liao JJ, Huang MC, Goetzl EJ. Cutting edge: alternative signaling of Th17cell development by sphingosine1-phosphate. J Immunol 2007; 178: 5425-5428.

8. Schett G, Gravallese E. Bone erosion in rheumatoid arthritis: mechanisms, diagnosis and treatment. Nat Rev Rheumatol 2012; 8: 656-664.

9. Cho ML, Jung YO, Kim KW, et al. IL-17 induces the production of IL-16 in rheumatoid arthritis. Exp Mol Med 2008; 40: 237-245.

10. He L, Hannon GJ. MicroRNAs: Small RNAs with a big role in gene regulation. Nat Rev Genet 2004; 5: 522-531.

11. Orellana EA, Kasinski AL. MicroRNAs in cancer: a historical perspective on the path from discovery to therapy. Cancers (Basel) 2015; 7: 1388-1405.

12.Almeida MI, Reis RM, Calin GA. MicroRNA history: discovery, recent applications, and next frontiers. Mutat Res 2011; 717: 1-8.

13. Hertel J, Lindemeyer M, Missal K, et al. The expansion of the metazoan microRNA repertoire. BMC Genomics 2006; 7: 25.

14. Kim HK, Lee YS, Sivaprasad U, et al. Muscle-specific microRNA miR-206 promotes muscle differentiation. J Cell Biol 2006; 174 677-687.

15. Haas JD, Nistala K, Petermann F, et al. Expression of miRNAs miR-133b and miR-206 in the $1117 \mathrm{a} / \mathrm{f}$ Locus Is Co-Regulated with IL-17 Production in $\alpha \beta$ and $\gamma \delta$ T Cells. PLoS ONE 2011; 6: e20171.

16. Pincus T, Yazici Y, Bergman M. Development of a multi-dimensional health assessment questionnaire (MD-HAQ) for the infrastructure of standard clinical care. Clin Exp Rheumatol 2005; 23: S19-S28.

17. Abo ElAtta AS, Ali YBM, Bassyouni IH, Talaat R. Upregulation of miR221/222 expression in rheumatoid arthritis (RA) patients: correlation with disease activity Clin Exp Med 2019; 19: 47-53.

18. Callis TE, Deng Z, Chen JF, et al. Muscling through the microRNA world. Exp Biol Med (Maywood) 2008; 233: 131-138.

19. Lee CT, Risom T, Strauss WM. Evolutionary conservation of microRNA regulatory circuits: an examination of microRNA gene complexity and conserved microRNA-target interactions through metazoan phylogeny. DNA Cell Biol 2007; 26: 209-218.
20.Gaur T, Hussain S, Mudhasani R, et al. Dicer inactivation in osteoprogenitor cells compromises fetal survival and bone formation, while excision in differentiated osteoblasts increases bone mass in the adult mouse. Dev Biol 2010; 340: 10-21.

21. Inose $\mathrm{H}$, Ochi $\mathrm{H}$, Kimura $A$, et al. microRNA regulatory mechanism of osteoblast differentiation. Proc Natl Acad Sci USA 2009; 106: 20794-20799.

22. Liu N, Williams AH, Maxeiner JM, et al. microRNA-206 promotes skeletal muscle regeneration and delays progression of Duchenne muscular dystrophy in mice. J Clin Invest 2012; 122: 2054-2065.

23. Zhao X, Xu D, Li Y, et al. MicroRNAs regulate bone metabolism. J Bone Miner Metab 2014; 32: 221-231.

24. Walsh NC, Reinwald S, Manning CA, et al. Osteoblast function is compromised at sites of focal bone erosion in inflammatory arthritis. J. Bone Miner Res 2009; 24: 1572-1585.

25. Sharma AR, Sharma G, Lee SS, et al. miRNA-Regulated Key Components of Cytokine Signaling Pathways and Inflammation in Rheumatoid Arthritis. Med Res Rev 2016; 36: 425-439.

26. Vicente R, Noël D, Pers YM, et al. Deregulation and therapeutic potential of microRNAs in arthritic diseases. Nat Rev Rheumatol 2016; 12: 211-220.

27. Xu S, Wang Y, Lu J, Xu J. Osteoprotegerin and RANKL in the pathogenesis of rheumatoid arthritis-induced osteoporosis. Rheumatol Int 2012; 32: 3397-3403.

28. Deng $\mathrm{X}, \mathrm{Su} \mathrm{Y}, \mathrm{Wu} \mathrm{H}$, et al. The role of microRNAs in autoimmune diseases with skin involvement. Scand J Immunol 2015; 81: 153-165.

29. van der Berg, Miossec P. IL-17 as a future therapeutic target for rheumatoid arthritis. Nat Rev Rheumatol 2009; 5: 549-553.

30. Adamopoulos IE, Chao CC, Geissler R, et al. Interleukin-17A upregulates receptor activator of NF-kappaB on osteoclast precursors. Arthritis Res Ther 2010; 12: R29.

31. Blaschke S, Schulz H, Schwarz G, et al. Interleukin 16 expression in relation to disease activity in rheumatoid arthritis. J Rheumatol 2001; 28: 12-21.

32. Klimiuk PA, Goronzy JJ, Weyand CM. IL-6 as an anti-inflammatory cytokine in rheumatoid synovitis. J Immunol 1999; 162: 4293-4299. 